\title{
Amplitude Distortion of Measured Leak Noise Signals caused by Instrumentation: Effects on Leak Detection in Water Pipes using the Cross-Correlation Method
}

M.J. Brennan ${ }^{1 *}$, Y. Gao ${ }^{2}$, P.C. Ayala ${ }^{1}$, F.C.L. Almeida ${ }^{3}$, P.F. Joseph ${ }^{4}$, A.T. Paschoalini ${ }^{1}$

${ }^{1}$ Department of Mechanical Engineering, UNESP, Ilha Solteira, SP15385-000, Brazil.

${ }^{2}$ Key Laboratory of Noise and Vibration Research, Institute of Acoustics, Chinese Academy of Sciences, Beijing, China.

${ }^{3}$ Faculty of Science and Engineering, UNESP, Tupã, SP 17602-496, Brazil

${ }^{4}$ Institute of Sound and Vibration Research, University of Southampton, Southampton, UK

* Corresponding author

No of pages: 28

No of figures: 9

No of tables: 0

14 August 2018 


\begin{abstract}
A common way to detect and locate leaks in buried water pipes is to use leak noise correlators. Vibration or acoustic signals are measured on the pipe using sensors placed either side of the leak, and the difference in the leak noise arrival times (time delay) at the sensors is estimated from the peak in the cross-correlation function of these signals. Over many years, much effort has been spent on improving the quality of the leak noise signals with the aim of improving the time delay estimate. In this paper it is shown that even if the signals suffer from severe amplitude distortion through either clipping or quantization, then an accurate time delay estimate can be obtained provided that the zero crossings in the noise data are preserved. This is demonstrated by using polarity co-incidence correlation on simulated and measured data. The use of random telegraph theory is also used as an approximation to allow the derivation of approximate analytical solutions for the cross-correlation function and cross spectral density of clipped noise to facilitate further insight into the effects of severe clipping.
\end{abstract}




\section{Introduction}

Since the early 1980 's leak noise correlators have been used to determine the existence and location of leaks in buried water pipes [1]. This method involves making leak noise measurements using two sensors that bracket the suspected leak position, sensing either acoustic pressure inside the pipe, or the velocity, or acceleration of a fitting on the pipe. The cross-correlation function of the two measured signals is then calculated, and the peak in this function indicates the difference in propagation times between the leak and the sensors. By combining this with knowledge of the speed at which the leak noise propagates, the location of the leak can be determined. The instrumentation (both hardware and software) has improved enormously since the first correlator was employed, and many contemporary leak noise correlators use the latest technology.

Although leak noise correlation has been extremely successful when used on metal pipes, since the widespread introduction of plastic pipes, a number of difficulties have emerged [2]. These include the relatively high rates of attenuation experienced by waves propagating along the pipes and the variability in the speed at which they propagate along the pipe, see for example [3-7], and the references therein. The poor performance of correlators for plastic pipes motivated some fundamental modelling work to determine the way in which a plastic pipe affects the cross-correlation function of leak noise signals [8,9]. Furthermore, the choice of sensor type for this type of pipe was also studied [10]. Using the model, and by comparing the results with several data sets from different countries, it was found that if the leak is close to one of the measurement positions, then the signal level at this position can be very large and is prone to clipping by the correlator instrumentation. At the other measurement position the signal can be very small and is prone to quantization in the analogue to digital conversion process. This poses 
the question as to what the effects of clipping and quantization, which cause amplitude distortion of the signals, are on the cross-correlation function and hence time delay estimation.

A seminal piece of work on the effects of clipping on a correlation function was carried out by Van Vleck during World War II [11]. The research involved the jamming of radar and communication systems, and was therefore classified. It was not until 1966 that the work appeared in the public domain [12]. In this paper they derived a key result which became known as the arcsine law. It was shown that provided the two signals to be correlated have a Gaussian distribution, then if there is severe clipping, such that the normalised signals $x_{1}$ and $x_{2}$ have a value of either 1 or -1 at any instant in time, then $\left.\rho_{x_{1} x_{2}}(\tau)\right|_{\text {clipped }}=2 / \pi \times \sin ^{-1}\left(\rho_{x_{1} x_{2}}(\tau)\right)$, where $\rho_{x_{1} x_{2}}(\tau)$ is the cross-correlation coefficient of the unclipped signals and $\left.\rho_{x_{1} x_{2}}(\tau)\right|_{\text {clipped }}$ is the cross-correlation coefficient of the clipped signals. This important result shows that the crosscorrelation of the actual signals can be reconstructed from the cross-correlation function of the severely clipped signals.

In the early 1960's, much work was conducted on the effects of quantization on correlation functions, for example [13-16]. In [13] Weiseb discussed the case of a one-bit quantizer (polarity co-incidence correlator), which has the same mathematical form as for the severe clipping case discussed by Van Vleck. Using Van Vleck's result, Weiseb showed that for Gaussian random signals, the upper bound on the rms deviation of the cross-correlation coefficient of data passed through the one-bit quantizer is only $\pi / 2$ times that of the corresponding crosscorrelation co-efficient of the unclipped data. Although this is small, it can be improved by 
increasing the sampling frequency and asymptotes to a value of 1.252 as the sampling frequency increases [17]. Thus, it can be seen that the effect of severe clipping has only a small effect on the cross-correlation function, and by sampling at a high frequency, the additional random noise added to the cross-correlation function by severe clipping can be reduced.

Using the expression for the error due to Gaussian random noise in time delay estimation calculated using the cross-correlation function $[18,19]$, Cusani $[20]$ showed that the error due to the polarity co-incidence correlator is also very small compared to the same correlation carried out with no quantization error. As the error for time delay estimation due to Gaussian random noise for leak detection using cross-correlation technique is likely to be small [9], the additional error due to severe clipping or extreme quantization is also likely to be small.

Motivated by the literature discussed above, the aim of this paper is to investigate the effects of the amplitude distortion of leak noise signals, through either severe clipping or extreme quantization caused by instrumentation, on time delay estimation for leak location in buried plastic water pipes. Both acoustic signals from hydrophones and vibration signals for accelerometers are considered. The physical causes of leak noise and the factors that affect its spectral content are not considered. Using the analytical model in $[8,10]$ and the Van Vleck Model $[11,12]$ some simulations are carried out to investigate the effects of severe clipping on the cross-correlation coefficient. An alternative approach to the analysis for heavily clipped signals is also considered using random telegraph theory [21,22]. Although this approach gives approximate results, it allows some closed-form expressions to be derived for both the crosscorrelation function (CCF) and Cross Power Spectral density (CPSD), which facilitates some physical insight into the effects of severe clipping in both simulations and experimental results. 
The paper is organised as follows. An overview of leak detection is given in Section 2. This is followed in Section 3, with a short discussion on the effects of signal distortion. Section 4 describes the analytical model of the plastic water pipe, and is followed by some simulations presented in Section 5, which uses the analytical model to generate measured leak noise signals. Section 6 investigates the effects of clipping on some experimental data, and Section 7 presents the alternative analysis using random telegraph signal theory. Finally, the paper is closed with some conclusions in Section 8.

\section{Overview of Leak Detection using Acoustic Correlation}

Figure 1 depicts a typical arrangement for water leak detection based on the cross-correlation of leak noise. Acoustic or vibration sensors are attached to convenient access points, either side of the suspected leak. The leak generates broadband noise, which propagates along the pipe, and the difference in the arrival times of the noise at the sensors (time delay) is used to determine the position of the leak. This is given by [8] $d_{1}=\left(d-c T_{0}\right) / 2$, where $c$ is the propagation speed of the leak noise (wave speed), which is mainly estimated from tables, but can also be measured in-situ [23] and $d$ is the distance between the sensors. The time delay $T_{0}$ is estimated from the peak in the CCF between the two measured signals $x_{1}(t)$ and $x_{2}(t)$, which is given by [8]

$$
R_{x_{1} x_{2}}(\tau)=\frac{1}{2 \pi} \int_{-\infty}^{+\infty} S_{x_{1} x_{2}}(\omega) e^{j \omega \tau} d \omega,
$$


where $j=\sqrt{-1}, S_{x_{1} x_{2}}(\omega)=\left|S_{x_{1} x_{2}}(\omega)\right| e^{j \phi_{x_{1} x_{2}}(\omega)}$ is the CPSD, in which $\left|S_{x_{1} x_{2}}(\omega)\right|$ and $\phi_{x_{1} x_{2}}(\omega)$ are the modulus and phase spectra between the signals $x_{1}(t)$ and $x_{2}(t)$ respectively, and $\omega$ is circular frequency. In the case when there is a pure time delay, then $\phi_{x_{1} x_{2}}(\omega)=-\omega T_{0}$.

\section{Signal Distortion - clipping and quantization}

As mentioned in the Introduction, at a measurement position, the amplitude of the leak noise can vary enormously, depending on the size of the leak and the distance of the measurement position from the leak. If a fixed gain measurement system is used, then two extreme situations are possible. The first is when the leak noise signal is larger than the dynamic range of the measurement system. In this case, clipping will occur. The second case is when the signal is very small compared to the dynamic range of the measurement system. In this case, the signal will be heavily quantized. If the most extreme case of clipping occurs, all amplitude information is lost, and the signal appears like a random telegraph signal [21,22], oscillating between two values corresponding to the maximum and minimum voltage levels of the instrumentation system. If the most extreme case of quantization occurs, then the signal will be represented by a single bit. In both these cases, the signal is only represented by two values. In this paper, to simulate these condtions, the leak noise signal is modified by the signum function, so that it takes a value of -1 if the signal is negative and +1 if the signal is positive. An example of a raw leak noise signal, and the same signal modified by the signum function is shown in Fig. 2. In the following sections, the effect of modifying a signal by the signum function on time delay estimation in plastic water pipes is discussed.

\section{Cross-Correlation Functions for a Buried Water Pipe}

A model of the CCF for a buried, water-filled, plastic pipe was first described in [8]. The CCF is given by Eq. (1), and the CPSD for pressure measurements is given by 
$S_{x_{1} x_{2}}(\omega)=H^{*}\left(\omega, d_{1}\right) H\left(\omega, d_{2}\right)$, where $H\left(\omega, d_{i}\right)=e^{-|\omega| \beta d_{i}} e^{-j \omega d_{i} / c}$, in which $\beta$ is a measure of the attenuation as the wave propagates along the pipe; $i=1$ or 2 depending on the measurement position, and the superscript $*$ denotes the complex conjugate. Thus

$$
S_{x_{1} x_{2}}^{(p)}(\omega)=e^{-|\omega| \beta d} e^{-j \omega T_{0}}
$$

where the superscript $(p)$ denotes pressure measurement, $T_{0}=\left(d_{2}-d_{1}\right) / c$, and the corresponding $\mathrm{CCF}$ is given by [8]

$$
R_{x_{1} x_{2}}^{(p)}(\tau)=\frac{\beta d}{\pi\left[(\beta d)^{2}+\left(\tau-T_{0}\right)^{2}\right]}
$$

In what follows, the superscript $(p)$ is used when the expressions are related to pressure measurements and the superscript $(a)$ is used to denote acceleration measurements. If there is no superscript, then the expression is valid for both sensor types. The autocorrelation function $R_{x_{i} x_{i}}^{(p)}(\tau)$ at distance $d_{i}$ from the leak position is given by

$$
R_{x_{i} x_{i}}^{(p)}(\tau)=\frac{2 \beta d_{i}}{\pi\left[\left(2 \beta d_{i}\right)^{2}+\tau^{2}\right]}
$$

Noting that the cross-correlation coefficient (CCC) is given by $\rho_{x_{1} x_{2}}(\tau)=R_{x_{1} x_{2}}(\tau) / \sqrt{R_{x_{1} x_{1}}(0) R_{x_{2} x_{2}}(0)}$, Eqs. (3) and (4) can be combined to give [8] 


$$
\rho_{x_{1} x_{2}}^{(p)}(\tau)=2 \frac{\sqrt{d_{1} d_{2}}}{d} \frac{(\beta d)^{2}}{(\beta d)^{2}+\left(\tau-T_{0}\right)^{2}}
$$

Applying the Fourier transform to the CCC, results in the normalised CPSD given by

$$
\hat{S}_{x_{1} x_{2}}^{(p)}(\omega)=2 \pi \beta \sqrt{d_{1} d_{2}} e^{-|\omega| \beta d} e^{-j \omega T_{0}},
$$

which should be compared with Eq. (2).

For a heavily clipped signal, or one where the Signum function has been applied, such as that shown in Fig. 2, there is a remarkably simple relationship between the cross-correlation coefficient and the cross-correlation coefficient of the unclipped signal. It is given by $[11,12]$,

$$
\left.\rho_{x_{1} x_{2}}(\tau)\right|_{\text {clipped }}=\frac{2}{\pi} \sin ^{-1}\left(\rho_{x_{1} x_{2}}(\tau)\right)
$$

To visualise the effect that severe clipping has on the $\mathrm{CCC}$, the quantity $\alpha=\left.\rho_{x_{1} x_{2}}(\tau)\right|_{\text {clipped }} / \rho_{x_{1} x_{2}}(\tau)$, is plotted in Fig. 3(a). It can be seen that for values of $\rho_{x_{1} x_{2}}(\tau)$ less than about 0.4 , the effect of severe clipping to give $\left.\rho_{x_{1} x_{2}}(\tau)\right|_{\text {clipped }}$, is simply to multiply $\rho_{x_{1} x_{2}}(\tau)$ by $2 / \pi$. For values greater than this, the multiplying factor varies between $2 / \pi$ and 1 . Thus, the general effect of severe clipping on the CCC is to distort its shape, giving a sharper peak. If the maximum value of $\rho_{x_{1} x_{2}}(\tau)$ is less than about 0.4 then there is negligible distortion of the CCC when severe clipping occurs. 
The maximum value of $\rho_{x_{1} x_{2}}(\tau)$ is dependent upon background noise and the position of the leak. In the noise-free case, if the leak is equidistant from the two measurement points, i.e., when $d_{1}=d / 2$, then the peak in the CCC is 1 . If the leak occurs at a measurement position so that $d_{1}=0$ or $d_{2}=0$ then the peak in the CCC is 0 . If $0 \leq d_{1} \leq d$, then $\max \left\{\rho_{x_{1} x_{2}}^{(p)}(\tau)\right\}=2 \sqrt{\hat{d}_{1}\left(1-\hat{d}_{1}\right)}$, where $\hat{d}_{1}=d_{1} / d$, which is plotted in Fig. 3(b) as a solid green line. The reason for this behaviour is because of the low-pass filtering effect of the sections of pipe either side of the leak. Thus, unless the leak is equidistant between the two measurement positions then the spectral characteristics of $x_{1}(t)$ and $x_{2}(t)$ are different. For a pressure measurement, it is clear that if the leak is very close to one of the measurement positions for example $\hat{d}_{1} \leq 0.05$ or $\hat{d}_{1} \geq 0.95$, then $\max \left\{\rho_{x_{1} x_{2}}^{(p)}(\tau)\right\}<0.4$ and so severe clipping practically has no effect on the shape of the CCC. The dashed red line in Fig. 3(b) is related to acceleration measurements which is discussed next.

The cross-correlation function between two accelerometer signals can be determined by multiplying Eq. (2) by $\omega^{4}$ to give

$$
S_{x_{1} x_{2}}^{(a)}(\omega)=A \omega^{4} e^{-|\omega| \beta d} e^{-j \omega T_{0}}
$$

where $A$ is a constant related to the properties of the pipe. Here it is arbitrarily set to 1 without loss of generalisation. The corresponding CCF can be determined by calculating the fourth derivative of Eq. (3) with respect to $\tau$ to give 


$$
R_{x_{1} x_{2}}^{(a)}(\tau)=\frac{24 \beta d}{\pi}\left(\frac{5\left(\tau-T_{0}\right)^{4}-10(\beta d)^{2}\left(\tau-T_{0}\right)^{2}+(\beta d)^{4}}{\left((\beta d)^{2}+\left(\tau-T_{0}\right)^{2}\right)^{5}}\right)
$$

The resulting $\mathrm{CCC}$ is given by

$$
\rho_{x_{1} x_{2}}^{(a)}(\tau)=\left(2 \frac{\sqrt{d_{1} d_{2}}}{d}\right)^{5}(\beta d)^{6}\left(\frac{5\left(\tau-T_{0}\right)^{4}-10(\beta d)^{2}\left(\tau-T_{0}\right)^{2}+(\beta d)^{4}}{\left((\beta d)^{2}+\left(\tau-T_{0}\right)^{2}\right)^{5}}\right)
$$

Applying the Fourier transform to Eq. (10) results in the normalised CPSD, which is given by

$$
\hat{S}_{x_{1} x_{2}}^{(a)}(\omega)=\frac{4}{3} \pi\left(\beta \sqrt{d_{1} d_{2}}\right)^{5} \omega^{4} e^{-|\omega| \beta d} e^{-j \omega T_{0}}
$$

To determine the CCC for heavily clipped acceleration signals, Eq. (7) can be applied in the same way as for pressure signals. The relationship between the severely clipped and the unclipped signal is thus the same as for the pressure signal. However, the relationship between the maximum value of $\rho_{x_{1} x_{2}}(\tau)$ and the position of the leak, is different for the two types of signals. It is given by $\max \left\{\rho_{x_{1} x_{2}}^{(a)}(\tau)\right\}=\left(2 \sqrt{\hat{d}_{1}\left(1-\hat{d}_{1}\right)}\right)^{5}$ for acceleration signals, which is plotted in Fig. 3(b) as a dashed red line. It is clear that when the leak is close to one of the measurement positions $\max \left\{\rho_{x_{1} x_{2}}^{(a)}(\tau)\right\}$ is much smaller than $\max \left\{\rho_{x_{1} x_{2}}^{(p)}(\tau)\right\}$. This is because the similarity between the sensor signals is less for acceleration signals than for pressure signals, which occurs because an accelerometer acts as a filter effectively amplifying the high frequency content and diminishing the low frequency leak noise energy at the measurement 
positions [10]. As discussed above, the effect of severe clipping on the $\mathrm{CCC}$ is greater when the $\max \left\{\rho_{x_{1} x_{2}}^{(p)}(\tau)\right\}$ is closer to unity. For most leak positions, the maximum of the CCC is much less for an acceleration signal compared to a pressure signal as shown in Fig. 3(b), and hence the effect of severe clipping will generally have less of an effect on the shape of the CCC of acceleration measured signals compared to pressure signals.

\section{Alternative representation of a clipped signal using random telegraph signal theory}

An alternative analysis of the clipped signals to treat them as random telegraph signals $[21,22]$. This analysis is useful, because some approximate analytical expressions can be derived for both the normalised CPSD and CCF. In this analysis, however, the CCF is normalised by its maximum value when $\tau=T_{0}$. so that its maximum value is 1 . It is also only valid when the leak is close to the mid-point between the measurement positions. Thus, this analysis can be used to study the shape of the CCF, which is significantly changed when the two leak noise signals are similar, but not the actual value of the CCF for severely clipped data.

With random telegraph theory, the signals are assumed to be Poisson point processes, oscillating between two levels, similar to the heavily clipped signal shown in Fig. 2. In this case, provided that the average number of zero crossings per unit time are similar in each signal, given approximately by $N$, the normalised CCF can be written in a way similar to that for the Power Spectral Density (PSD) in [21,22] and is given by

$$
\left.\hat{R}_{x_{1} x_{2}}(\hat{\tau})\right|_{\text {telegraph }}=e^{-2 N\left|\hat{\tau}-\hat{T}_{0}\right|}
$$


where $\hat{\tau}=\tau / \beta d$ and $\hat{T}_{0}=T_{0} / \beta d$. Now, the average number of zero crossings for two sufficiently similar severely clipped Gaussian leak noise signals per unit time, derived by applying the signum function to the original signals, is the same as for the original Gaussian noise signals, which is given by [28]

$$
N=\frac{1}{\pi} \sqrt{-\left.\frac{d^{2}\left(\tilde{R}_{x_{1} x_{2}}(\hat{\tau})\right)}{d \hat{\tau}^{2}}\right|_{\hat{\tau}=\hat{T}_{0}}}
$$

where $\tilde{R}_{x_{1} x_{2}}(\hat{\tau})$ is the CCF of the measured signals.

\subsection{Pressure}

In Eq. (13), $\tilde{R}_{x_{1} x_{2}}(\hat{\tau})$ is set to $R_{x_{1} x_{2}}^{(p)}(\tau)$ for the unclipped pressure data from Eq. (3) with $\tau=\hat{\tau} \beta d$ and then divided by the maximum value of $R_{x_{1} x_{2}}^{(p)}(\tau)=1 / \pi \beta d$, to give

$$
\tilde{R}_{x_{1} x_{2}}^{(p)}(\hat{\tau})=\frac{1}{1+\left(\hat{\tau}-\hat{T}_{0}\right)^{2}}
$$

Differentiating Eq. (14) twice with respect to $\hat{\tau}$, evaluating the result at $\hat{\tau}=\hat{T}_{0}$ and substituting this into Eq. (13), gives $N=\sqrt{2} / \pi$. Combining this with Eq. (12) results in

$$
\left.\tilde{R}_{x_{1} x_{2}}^{(p)}(\hat{\tau})\right|_{\text {telegraph }}=e^{-\frac{2 \sqrt{2}}{\pi}\left|\hat{\tau}-\hat{T}_{0}\right|}
$$


The corresponding normalised CPSD can be determined by applying the Fourier transform to Eq. (15) to give

$$
\left.\tilde{S}_{x_{1} x_{2}}^{(p)}(\hat{\omega})\right|_{\text {telegraph }}=\frac{1}{\sqrt{2}\left(1+\frac{\hat{\omega}^{2} \pi^{2}}{8}\right)} e^{-j \hat{\omega} \hat{T}_{0}}
$$

where $\hat{\omega}=\omega \beta d$. There is no explicit analytical solution for the normalised CCF of clipped data, but it can be determined by combining Eqs. (7) and (14) to give

$$
\left.\tilde{R}_{x_{1} x_{2}}^{(p)}(\hat{\tau})\right|_{\text {clipped }}=\frac{2}{\pi} \sin ^{-1}\left(\frac{1}{1+\left(\hat{\tau}-\hat{T}_{0}\right)^{2}}\right)
$$

The corresponding CPSD can be determined numerically by applying the Fourier transform to Eq. (17) to give $\left.\tilde{S}_{x_{1} x_{2}}^{(p)}(\hat{\omega})\right|_{\text {clipped }}$. To visualise the difference between $\left.\tilde{S}_{x_{1} x_{2}}^{(p)}(\hat{\omega})\right|_{\text {telegraph }}$ and $\left.\tilde{S}_{x_{1} x_{2}}^{(p)}(\hat{\omega})\right|_{\text {clipped }}$ they are plotted in Fig. 4(ai), setting $\hat{T}_{0}=0$. Also overlaid in this plot is the exact normalised version of the CPSD for the unclipped data determined from Eq. (2) and given by $\tilde{S}_{x_{1} x_{2}}^{(p)}(\hat{\omega})=e^{-|\hat{\omega}|}$. It can be seen that the exact solution for the normalised CPSD for the clipped data, and that determined using telegraph signal theory are very similar. Examining the expression determined using telegraph signal theory given in Eq. (16), it can be seen that at high frequencies the CPSD is approximately $2^{\frac{5}{2}} / \hat{\omega}^{2} \pi^{2}$, which is much larger than $e^{-|\hat{\omega}|}$. The difference between these is the high frequency noise added to the data by severe clipping. 
The normalised CCF determined using telegraph signal theory, given by Eq. (15), and the exact solution for the clipped data given in Eq. (17) are plotted in Fig. 4(bi). Also plotted in this figure is the normalised CCF for the unclipped data given by Eq. (14). It can be seen that there are differences between the approximate and exact solutions for the clipped data, but they have a similar characteristic when $\hat{\tau} \approx \hat{T}_{0}$, which in this case shown is when $\hat{\tau} \approx 0$. In both cases for the severely clipped data the peak is considerably sharpened compared to the normalised CCF for the unclipped data.

To determine the similarity between the approximate and actual solutions for the normalised CCF, some approximations are made in the expressions for when $\left|\hat{\tau}-\hat{T}_{0}\right|<<1$. In this case Eq. (15) becomes

$$
\left.\tilde{R}_{x_{1} x_{2}}^{(p)}(\hat{\tau})\right|_{\text {telegraph }} \approx 1-\frac{2 \sqrt{2}}{\pi}\left|\hat{\tau}-\hat{T}_{0}\right|
$$

Using a geometrical interpretation, Eq. (17) can be written as

$$
\frac{\pi}{2}-\left.\frac{\pi}{2} \tilde{R}_{x_{1} x_{2}}^{(p)}(\hat{\tau})\right|_{\text {clipped }}=\sin ^{-1}\left(\frac{\left|\hat{\tau}-\hat{T}_{0}\right|\left(2+\left(\hat{\tau}-\hat{T}_{0}\right)^{\frac{1}{2}}\right)}{1+\left(\hat{\tau}-\hat{T}_{0}\right)^{2}}\right)
$$

Applying the condition $\left|\hat{\tau}-\hat{T}_{0}\right|<<1$, Eq. (19) can be simplified and rearranged to give 


$$
\left.\hat{R}_{x_{1} x_{2}}^{(p)}(\tau)\right|_{\text {clipped }} \approx 1-\frac{2 \sqrt{2}}{\pi}\left|\hat{\tau}-\hat{T}_{0}\right|,
$$

which is the same as Eq. (18). This shows that when $\hat{\tau} \approx \hat{T}_{0}$ the CCF given by the telegraph signal is similar to the exact solution for the normalised severely clipped signal. More importantly, the time delay information is preserved, even though the amplitudes of the signals are severely distorted.

\subsection{Acceleration}

In a similar way to the formulation for pressure, a normalised form of Eq. (9) can be determined by dividing by the maximum value of the CCF and setting $\tau=\hat{\tau} \beta d$. It is given by

$$
\tilde{R}_{x_{1} x_{2}}^{(a)}(\hat{\tau})=\frac{5\left(\hat{\tau}-\hat{T}_{0}\right)^{4}-10\left(\hat{\tau}-\hat{T}_{0}\right)^{2}+1}{\left(1+\left(\hat{\tau}-\hat{T}_{0}\right)^{2}\right)^{5}}
$$

Differentiating Eq. (21) twice with respect to $\hat{\tau}$, evaluating the result at $\hat{\tau}=\hat{T}_{0}$ and substituting this into Eq. (13), gives $N=\sqrt{30} / \pi$. Combining this with Eq. (12) results in

$$
\left.\tilde{R}_{x_{1} x_{2}}^{(a)}(\hat{\tau})\right|_{\text {telegraph }}=e^{-\frac{2 \sqrt{30}}{\pi}\left|\hat{t}-\hat{T}_{0}\right|}
$$

The corresponding normalised CPSD can be determined by applying the Fourier transform to Eq. (22) to give 


$$
\left.\tilde{S}_{x_{1} x_{2}}^{(a)}(\hat{\omega})\right|_{\text {telegraph }}=\frac{1}{\sqrt{30}\left(1+\frac{\hat{\omega}^{2} \pi^{2}}{120}\right)} e^{-j \hat{\omega} \hat{T}_{0}}
$$

Again, there is no explicit analytical solution for the normalised CCF of clipped data, but it can be determined by combining Eqs. (7) and (21) to give

$$
\left.\tilde{R}_{x_{1} x_{2}}^{(a)}(\hat{\tau})\right|_{\text {clipped }}=\frac{2}{\pi} \sin ^{-1}\left(\frac{5\left(\hat{\tau}-\hat{T}_{0}\right)^{4}-10\left(\hat{\tau}-\hat{T}_{0}\right)^{2}+1}{\left(1+\left(\hat{\tau}-\hat{T}_{0}\right)^{2}\right)^{5}}\right)
$$

The corresponding CPSD can be determined numerically by applying the Fourier transform to Eq. (24) to give $\left.\tilde{S}_{x_{1} x_{2}}^{(a)}(\hat{\omega})\right|_{\text {clipped }}$. To visualise the difference between $\left.\tilde{S}_{x_{1} x_{2}}^{(a)}(\hat{\omega})\right|_{\text {telegraph }}$ and $\left.\tilde{S}_{x_{1} x_{2}}^{(a)}(\hat{\omega})\right|_{\text {clipped }}$ they are plotted in Fig. 4(aii), setting $\hat{T}_{0}=0$. Also overlaid in this plot is the exact normalised version of the CPSD for the unclipped data determined from Eq. (8) and given by $\tilde{S}_{x_{1} x_{2}}^{(a)}(\hat{\omega})=(\beta d)^{4} \times S_{x_{1} x_{2}}^{(a)}(\hat{\omega})=\hat{\omega}^{4} e^{-|\hat{\omega}|}$. It can be seen that the exact solution for the normalised CPSD for the clipped data, and that determined using telegraph signal theory are very similar at high frequencies even though there are considerable differences at low frequencies. Examining the expression determined using telegraph signal theory given in Eq. (16), it can be seen that at high frequencies the CPSD is approximately $\left.\tilde{S}_{x_{1} x_{2}}^{(a)}(\hat{\omega})\right|_{\text {telegraph }} \approx 4 \sqrt{30} / \hat{\omega}^{2} \pi^{2}$. This is proportional to $1 / \hat{\omega}^{2}$, as with the pressure measurements, and is much larger than $\hat{\omega}^{4} e^{-|\hat{\omega}|}$

The normalised CCF determined using telegraph signal theory, given by Eq. (23), and the exact solution for the clipped data given in Eq. (24) are plotted in Fig. 4(bii). Also plotted in this 
figure is the normalised CCF for the unclipped data given by Eq. (21). As with the pressure measurements, it can be seen that there are some differences between the approximate and exact solutions for the clipped data, but they have a similar characteristic when $\hat{\tau} \approx \hat{T}_{0}$, which in this case shown is when $\hat{\tau} \approx 0$. They are also similar to the normalised CCF for the unclipped acceleration data as well, and when $\left|\hat{\tau}-\hat{T}_{0}\right|<<1$ then

$$
\left.\tilde{R}_{x_{1} x_{2}}^{(a)}(\hat{\tau})\right|_{\text {telegraph }} \approx 1-\frac{2 \sqrt{30}}{\pi}\left|\hat{\tau}-\hat{T}_{0}\right|
$$

This analysis shows that the peak in the acceleration CCF is sharper than that for the pressure data, and this is due to the higher frequency content in this data because of the filtering effect of the accelerometers. Moreover, it can be seen that close to the peak in the CCF, the effect of severe clipping for acceleration data is very small. As with pressure data, the time delay information is preserved, even though the amplitudes of the signals are severely distorted.

\section{Numerical Simulations}

\subsection{Simulations}

Simulations are carried out to compare numerical results with analytical predictions given in Section 4. A diagram showing the way in which leak noise signals are generated is shown in Fig. 5. The leak is assumed to generate Gaussian white noise. Although this is not strictly true in practice as leak noise at source has limited bandwidth [24], it is considered to be an acceptable assumption for the purposes of this paper. The noise propagates through a section of pipe with length $d_{1}$ and frequency response function (FRF) $H_{1}(\omega)=e^{-\omega \beta d_{1}} e^{-j \omega d_{1} / c}$ to pressure sensor 1, and through a section of pipe with length $d_{2}$ and FRF $H_{2}(\omega)=e^{-\omega \beta d_{2}} e^{-j \omega d_{2} / c}$ to pressure sensor 2 . Thus, the pressure time histories can be easily calculated by either 
convolution of the corresponding impulse responses of $H_{1}(\omega)$ and $H_{2}(\omega)$ with the time history of the leak noise, or by multiplying the spectrum of the leak noise with the FRFs $H_{1}(\omega)$ and $H_{2}(\omega)$, and then using the inverse Fourier transform. To simulate the severely clipped signals, the signum function is applied to the pressure signal time histories at the measurement positions. This operation is denoted by SF in Fig. 4. To simulate acceleration signals, the FRFs $H_{1}(\omega)$ and $H_{2}(\omega)$, of the pressure signals are simply multiplied by $-\omega^{2}$, and the severely clipped acceleration signals are again determined using the signum fiunction.

A specific pipe system was chosen for simulations, which is a test rig in Canada [2], from which experimental data is processed and presented in Section 5. The pipe has a value of $\beta=1.15 \times 10^{-4} \mathrm{~s} / \mathrm{m}$ and a wave speed of $c=475 \mathrm{~m} / \mathrm{s}$, which were determined by matching the model for pressure signals to experimental results. Before any time-histories are processed they are normalised so that they have zero mean and a standard deviation of unity. The length between the sensors is set to $100 \mathrm{~m}$ (which is not precisely the same as the actual test rig), and three leak positions are considered: $60 \mathrm{~m}, 70 \mathrm{~m}$ and $90 \mathrm{~m}$. For each leak position, four graphs are plotted. They are the modulus and phase of the CPSD, the coherence between the signals and the CCC. Furthermore, data are presented for severely clipped and unclipped signals for both pressure and acceleration signals. The graphs for pressure signals are plotted in Fig. 6 and the graphs for acceleration signals are plotted in Fig. 7.

With the exception of the coherence function, four quantities are plotted in each subplot in Figs. 6 and 7. They are the theoretical values for the severely clipped and unclipped signals, and those calculated from the random time series data. For the pressure data, the theoretical modulus and phase of the CPSD of the unclipped signals are plotted using Eq. (2), the corresponding plots for the severely clipped signals, are calculated by combining Eqs. (5) and 
(7), and then using the Fourier transform. For the acceleration data, the theoretical modulus and phase of the CPSD of the unclipped signals are plotted using Eq. (8), the corresponding plots for the severely clipped signals, are calculated by combining Eqs. (7) and (10), and then using the Fourier transform. The two other quantities plotted are the values corresponding to the unclipped and severely clipped data calculated numerically as discussed above and illustrated in Fig. 4. The sampling frequency for both the 60 second-long pressure and acceleration time histories is $4 \mathrm{kHz}$. The transformation to the frequency domain is carried out using a 4096 point FFT and a Hanning window with 50\% overlap.

\subsection{Discussion}

\subsubsection{Pressure Data}

Examining the modulus of the CPSD for all three measurement positions, the low-pass filtering property of the pipe for the unclipped data is evident. Note that this is independent of the position of the leak but is a function of the distance between the sensors, because from Eq. (2) $\left|S_{x_{1} x_{2}}^{(p)}(\omega)\right|=e^{-|\omega| \beta d}$. Note also, that the analytical and numerical results for unclipped modulus data are practically the same for all three cases. The addition of high frequency noise due to severe clipping is clear. Consider first the analytical results. It can be seen that clipping has a greater effect when the distances from the leak to the measurement positions are similar, and has a diminishing effect when these distances are very different, such as when $d_{1}=90 \mathrm{~m}$ and $d_{2}=10 \mathrm{~m}$. The reason for this is because when the distances are very different, for example when $\hat{d}_{1}<<1$, the maximum value of the CCF is small, as shown in Fig. 3(b). When this occurs, then $\left.\rho_{x_{1} x_{2}}(\tau)\right|_{\text {clipped }} / \rho_{x_{1} x_{2}}(\tau) \approx 2 / \pi$ as shown in Fig. 3(a), so there is very little distortion in the shape of the CCC, and so there is very little distortion in the CPSD due to clipping. The reason why the noise in the numerical data does not follow the same pattern is because the coherence 
between the two leak noise signals becomes increasingly poor at high frequencies as can be seen in Figs 6(ci-ciii). This means that the estimate of the CPSD becomes increasingly poor. Examining Figs. 6(bi-biii), it can be seen that the slope of the unwrapped phase increases as the relative distance between the leak and the measurement positions increases. It can also be seen that as this relative distance increases, the frequency beyond which it is not possible to unwrap the data decreases, and this is related to the coherence between the measured pressure signals [25]. Finally, it can be seen that apart from the unwrapping issue, the effect of severe clipping has no effect on the phase spectrum.

Concerning the CCCs shown in Figs. 6(di-diii), it can be seen that the effect of severe clipping has no impact on the time delay estimation. Its main effect is to reduce the maximum value, as the relative distance between the leak and the measurement positions increases, as shown in Fig. 3(a). There is a small change in the shape of the CCC, especially when the relative distance between the leak and the measurement positions is small. The main effect of severe clipping, in this case, is to sharpen the peak. Note, that there is a negligible difference between the analytical solutions and the numerical solutions for both the unclipped and severely clipped signals, for any of the three measurements.

\subsubsection{Acceleration Data}

The processed acceleration data is shown in Fig. 7 in the same format as for the pressure data in Fig. 6. Examining the modulus of the CPSD in Figs. 7(ai-aiii), it can be seen that the main difference of the unclipped and severely clipped acceleration data compared to the corresponding pressure data, is that the low frequencies have been supressed and the high frequencies have been amplified. This is because of the differential effect of the accelerometer

so that $\left|S_{x_{1} x_{2}}^{(a)}(\omega)\right|=A \omega^{4}\left|S_{x_{1} x_{2}}^{(p)}(\omega)\right|$, which can be seen from Eqs. (2) and (8) [10,]. The 
combination of the pipe and the accelerometer forms a band-pass filter $[5,10]$ with the peak in the modulus of the CPSD occurring at a frequency of $\left.f\right|_{\text {peak }}=2 /(\pi \beta d)$ [26]. Because the coherence is poor at low frequencies, as well as at high frequencies due to the filtering effect of the accelerometers as observed in Figs. 7(ai-aiii), the noise is evident in the modulus of the numerically evaluated CPSDs at low and high frequencies for the severely clipped data, for all three measurement positions. Examining the phase data, it can be seen that they are identical to the pressure data, with the exception of the numerical solutions for the severely clipped data, in which it is clear that the frequency at which the unwrapping fails is higher, because of the amplifying effects of the accelerometer at high frequencies.

Examining the CCCs shown in Figs. 7(di-diii), it can be seen that the use of an accelerometer sharpens the peak considerably, and the maximum value reduces sharply as the relative distance between the leak and the measurement positions increases as indicated by Fig. 3(a). The effect of severe clipping has no impact on the time delay estimation, and only has a small effect on the shape of the CCC in all cases. Note that, as with the pressure data, there is a negligible difference between the analytical solutions and the numerical solutions for both the unclipped and severely clipped signals, for all three measurements.

\section{Field measurements}

Measured signals from a pipe rig, specially constructed for water leak detection at the National Research Council campus in Canada, are used to illustrate the effects of severe clipping on actual leak noise data. The description of the test site and measurement procedures are given in $[2,8]$, and a schematic of the site is shown in Fig. 8(a). Noise from a leak, an illustration of which is shown in Fig. 8(b), was measured using hydrophones and accelerometers at two access points, one of which is shown in Fig. 8(c). The distance between the measurement points was 
102.6 $\mathrm{m}$, and the distance of the upstream measurement point from the leak was $73.5 \mathrm{~m}$. The signals of $66 \mathrm{~s}$ duration were each passed through an anti-aliasing filter with the cut-off frequency set at $200 \mathrm{~Hz}$ and then sampled at a frequency of $500 \mathrm{~Hz}$. The frequency domain data were determined using a 1024-point FFT, a Hanning window, with 50\% overlap, and spectral averaging. The results are shown in Fig. 9 for both unclipped and severely clipped pressure and acceleration data, using the same format as with the simulations. Also shown in the figure, are predictions calculated using the analytical model. Severe clipping of the measured data was simulated by using the signum function, in the same way as for the simulated data.

\subsection{Pressure data}

First, examining the modulus of CPSD for the pressure data shown in Fig. 9(ai), it can be seen that the experimental results follow the predictions up to about $50 \mathrm{~Hz}$, and then they deviate at higher frequencies. The reason for the deviation is due to a resonance in the pipe-sensor system as discussed in [27]. The severely clipped data and the predictions match very well over the whole frequency range, and show that the effect of clipping is to add noise to the leak noise data, above about $50 \mathrm{~Hz}$. Concerning the phase, shown in Fig. 9(bi), it can be seen that the phase is successfully unwrapped up to around $50 \mathrm{~Hz}$ for both unclipped and severely clipped data, even though the coherence is significantly reduced when the data is severely clipped, as shown in Fig. 9(ci). To calculate the CCC the data are first passed through a band-pass filter with lower and upper cut-off frequencies set to 10 and $50 \mathrm{~Hz}$ respectively to remove unwanted background noise. The CCC for the pressure data is plotted in Fig. 9(di). Note that, unlike the simulations shown in the previous section, the CCC for the severely clipped data is multiplied by $\pi / 2$ so that the shapes of the CCC for the unclipped and severely clipped data can be easily compared. It can be seen that severe clipping of the data does not change the estimated time 
delay or the shape of the CCC (in the figure the plots are indistinguishable). The reason for this is the same as discussed in the previous section for simulated data. The differences between the shape of the CCC for the predictions and the measurements can be attributed to unmodeled noise. Note, however, that the shape of the predicted and measured CCCs close to the estimated time delay of $92 \mathrm{~ms}$ are very similar.

\subsection{Acceleration data}

First, consider the modulus of CPSD for the acceleration data shown in Fig. 9(aii). The bandpass characteristic is evident, as discussed for the simulated data. The effect of measuring acceleration rather than pressure is to increase the frequency range over which the leak data can be processed, and it is clear that there is very little difference in the modulus of the CPSD for the unclipped and the severely clipped data. In this particular pipe system there are no resonances evident in the acceleration data unlike with the pressure data, as discussed in [27], and this means that the phase can be unwrapped up to a much higher frequency than for the pressure data - just over $100 \mathrm{~Hz}$ compared with about $50 \mathrm{~Hz}$. However, note in Fig. 9(bii) that the effect of severe clipping is to reduce the frequency at which the phase can be unwrapped. In Fig. 9(cii), it can be seen that the coherence is much smaller than for pressure data, for unclipped data, and is similar for severely clipped data. Finally, for the CCC plotted in Fig. 9(dii), it can be seen that, as with the pressure data, severe clipping of the data does not change the estimated time delay or the shape of the CCC (in the figure the plots are indistinguishable). Note that the CCC for the severely clipped data is multiplied by $\pi / 2$, as with the pressure data. The narrow peak in the CCC for the acceleration data compared with the pressure data, is due to the wider bandwidth of the acceleration data. 


\section{Conclusions}

This paper has described an analytical, numerical and experimental study into the effects of severe amplitude distortion of leak noise data due to either severe clipping or extreme quantization caused by instrumentation which is part of a leak noise correlator. The efficacy of using cross-correlation on severely clipped signals for time delay estimation in the location of leaks in buried plastic water distribution pipes has been investigated. Two types of data have been analysed, namely acoustic pressure inside the pipe, and acceleration of the pipe wall. To study severe clipping of the data, the signum function was applied to the data removing all amplitude information, effectively keeping only the zero crossings in the signals. Applying Van Vleck's result for severely clipped signals, it was shown that severe clipping has a greater effect on pressure data than acceleration data. Moreover, it has a greater effect if the measured leak signals are similar, such that the peak in the CCC is close to unity. If this is not the case, which can occur either when the leak is close to one of the measurement positions or when the signal to noise ratio is small, then the effect of severe clipping on the shape of the CCC function is negligible. To gain some additional insight into the effect of severe clipping, random telegraph theory has been applied to the theoretical model of the $\mathrm{CCF}$ for leak noise from plastic water pipes. New analytical expressions have been presented for the normalised CCFs and the corresponding CPSDs for pressure and acceleration measurements. These expressions allow insight into the shape of the CCF at time delays close to the peak, and also the behaviour of the CPSD at high frequencies.

Generally, it has been shown that the effect of severe clipping does not have an appreciable effect on the normalised CCF and hence the location of the leak. Its main effect is to add high frequency correlated noise to the data, which does not have detrimental effect on time delay estimation. In the experimental pressure and acceleration data presented, the effect of severe 
clipping was barely discernible in the normalised CCF. This result has a major implication for the development of leak noise correlators. It means that electronic circuits for automatic gain control are unnecessary, as distortion of the leak noise signals by clipping has a negligibly small effect on time delay estimation. Furthermore, the fidelity of the digitised signals using a large number of bits in the ADC is also unnecessary. If the leak noise is at least as large as the voltage level of a single bit, then the time delay estimation using two leak noise signals is possible.

\section{Acknowledgements}

The authors gratefully acknowledge the National Research Council in Canada for providing the leak noise test data. Professor Gao would like to acknowledge the financial support of the Natural Science Foundation of China (under Grant 11774378). The remaining authors would like to acknowledge the financial support of FAPESP (Process No. 2013/50412-3). Professor Brennan would further like to acknowledge the financial support of FAPESP (Process Nos. 2018/04407-1 and 2018/25360-3). 


\section{References}

[1] H.V. Fuchs, R. Riehle, Ten years of experience with leak detection by acoustic signal analysis, Applied Acoustics, 33, (1991), 1-19.

[2] O. Hunaidi, W.T. Chu, Acoustical characteristics of leak signals in plastic water distribution pipes. Applied Acoustics, 58(3), (1999), 235-254.

[3]. J.M. Muggleton, M.J. Brennan, R.J. Pinnington, Wavenumber prediction in buried pipes for water leak detection. Journal of Sound and Vibration, 249(5), (2002), 939-954.

[4]. J.M. Muggleton, M.J. Brennan, P.W. Linford, Axisymmetric wave propagation in fluid-filled pipes: Measurements in in-vacuo and buried pipes. Journal of Sound and Vibration, 270, (2004), 171-190.

[5]. F.C.L. Almeida, M.J. Brennan, P.F. Joseph, S. Whitfield, S. Dray, A. Paschoalini, On the acoustic filtering of the pipe and sensor in a buried plastic water pipe and its effect on leak detection: An experimental investigation. Sensors, 14, (2014), 5595-5610.

[6] Y. Gao, Y. Liu, J.M. Muggleton, Axisymmetric fluid-dominated wave in fluid-filled plastic pipes: loading effects of surrounding elastic medium. Applied Acoustics, 116, (2017), 43-49.

[7] M.J. Brennan, M. Karimi, J.M. Muggleton, F.C.L. Almeida, F. Kroll de Lima, P.C. Ayala, D. Obata, A.T. Paschoalini, N. Kessissoglou, On the effects of soil properties on leak noise propagation in plastic water distribution pipes, Journal of Sound and Vibration, 427, (2018), 120-133.

[8] Y. Gao, M.J. Brennan, P.F. Joseph, J.M. Muggleton, O, Hunaidi, A model of the correlation function of leak noise in buried plastic pipes. Journal of Sound and Vibration, 277, (2004), 133-148.

[9] Y. Gao, M.J. Brennan, P.F. Joseph, A comparison of time delay estimators for the detection of leak noise signals in plastic water distribution pipes. Journal of Sound and Vibration, 292, (2006), 552-570.

[10] Y. Gao, M.J. Brennan, P.F. Joseph, J.M. Muggleton, O, Hunaidi, On the selection of acoustic/vibration sensors for leak detection in plastic water pipes. Journal of Sound and Vibration, 283, (2005), 927-941.

[11] J.H. Van Vleck, The spectrum of clipped noise, Radio Research Laboratory Research Report 51, Harvard University, Cambridge, Massachusetts, USA, (1943).

[12] J.H. Van Vleck, D. Middleton, The spectrum of clipped noise, Proceedings of the IEEE, 54, 1, (1966), 2-19.

[13] S. Weinreb. A digital spectral analysis technique and its application to radio astronomy. Massachusetts Institute of Technology Technical Report 412, (1963), 119 pages. 
[14] D.G. Watts, A general theory of amplitude quantization with applications to correlation determination. Proceedings of the IEE - Part C: Monographs, 9(15), (1962), 209-2018.

[15] B.F.C. Cooper, Correlators with two-bit quantization, Australian Journal of Physics, 23, (1970), 521-527.

[16] J.R. Jordan, Correlation algorithms, circuits and measurement applications, Proceedings of the IEE - Part C: Electronic Circuits and Systems, 133(1), (1986), 5874.

[17] F.K. Bowers, R.J. Klingler, Quantization noise of correlation spectrometers, Astronomy and Astrophysics Supplement, 15, (1974), 373-380.

[18] G.C. Carter, Ph.D. Dissertation: Time delay estimation. University of Connecticut, 1976.

[19] C.H. Knapp, G.C. Carter, The generalised correlation method for estimation of time delay, IEEE Transactions on Acoustics, Speech, and Signal Processing, 24(4), (1976) 320-327.

[20] R. Cusani, Fast techniques for time delay estimation, Proceedings of the Electrotechnical Conference Integrating Research, Industry and Education in Energy and Communication Engineering, 11-13 April 1989, Lisbon, Portugal, (1989), 177180.

[21] J.S. Bendat, A.G. Piersol, Random data analysis and measurement procedures, $3^{\text {rd }}$ Edition, John Wiley and Sons. Inc, New York, (2000).

[22] K. Shin, J.K. Hammond, Fundamentals of Signal Processing for Sound and Vibration Engineers, John Wiley, Chichester, (2008).

[23] F.C.L. Almeida, M.J. Brennan, P.F. Joseph, S. Dray, S. Whitfield, A.T. Paschoalini, Towards an in-situ measurement of wave velocity in buried plastic water distribution pipes for the purposes of leak location. Journal of Sound and Vibration, 359, (2015), $40-55$.

[24] A.S. Papastefanou, P.F. Joseph, M.J. Brennan, Experimental Investigation into the Characteristics of In-Pipe Leak Noise in Plastic Water Filled Pipes. Acta Acustica united with Acustica, 98(6), (2012), 847-856.

[25] J.M. Muggleton, M.J. Brennan, Y. Gao, Determining the location of buried plastic water pipes from measurements of ground surface vibration, Journal of Applied Geophysics, 75(1), (2011), 54-61.

[26] M.J. Brennan, F. Kroll de Lima, F.C.L. Almeida, P.F. Joseph, A.T. Paschoalini, A virtual pipe rig for testing acoustic leak detection correlators: Proof of concept, Applied Acoustics, 102, (2016), 137-145. 
[27] F.C.L. Almeida, M.J. Brennan, P.F. Joseph, Y. Gao, A.T. Paschoalini, The effects of resonances on time delay estimation for water leak detection in plastic pipes. Journal of Sound and Vibration, 420, (2018), 315-329.

[28] S.O. Rice, The mathematical analysis of random noise, The Bell System Technical Journal 23(3), (1944), 282-232. 